\title{
The expression of cytoglobin as a prognostic factor in gliomas: a retrospective analysis of 88 patients
}

\author{
Hong-Wu Xu ${ }^{1,2}$, Yue-Jun Huang ${ }^{2,3}$, Ze-Yu Xie ${ }^{1 *}$, Lan Lin ${ }^{1}$, Yan-Chun Guo ${ }^{1}$, Ze-Rui Zhuang ${ }^{1}$, Xin-Peng Lin ${ }^{1}$, \\ Wen Zhou' ${ }^{1}$, Mu Li ${ }^{1}$, Hai-Hua Huang ${ }^{4}$, Xiao-Long Wei ${ }^{5}$, Kwan Man ${ }^{6}$ and Guo-Jun Zhang ${ }^{7^{*}}$
}

\begin{abstract}
Background: Evidence suggests that cytoglobin (Cygb) may function as a tumor suppressor gene.

Methods: We immunohistochemically evaluated the expression of Cygb, phosphatidylinositol-3 kinase (PI-3K), phosphorylated (p)-Akt, Interleukin-6 (IL-6), tumor necrosis factor-a (TNFa) and vascular endothelial growth factor (VEGF) in 88 patients with 41 high-grade gliomas and 47 low-grade gliomas. Intratumoral microvessel density (IMD) was also determined and associated with clinicopathological factors.

Results: Low expression of Cygb was significantly associated with the higher histological grading and tumor recurrence. A significant negative correlation emerged between Cygb expression and PI3K, p-Akt, IL-6, TNFa or VEGF expression. Cygb expression was negatively correlated with IMD. There was a positive correlation between PI3K, p-Akt, IL-6, TNFa and VEGF expression with IMD.High histologic grade, tumor recurrence, decreased Cygb expression, increased PI3K expression, increased p-Akt expression and increased VEGF expression correlated with patients' overall survival in univariate analysis. However, only histological grading and Cygb expression exhibited a relationship with survival of patients as independent prognostic factors of glioma by multivariate analysis.
\end{abstract}

Conclusions: Cygb loss may contribute to tumor recurrence and a worse prognosis in gliomas. Cygb may serve as an independent predictive factor for prognosis of glioma patients.

Keywords: Glioma, Cytoglobin, Phosphatidylinositol-3 kinase, Recurrence, Prognosis

\section{Background}

Glioma is the most common brain tumor in adults. Its ability to evade immune surveillance and impede antitumor responses leads to sustained growth and enhanced malignancy [1,2]. Increasing evidence indicates that cytoglobin (Cygb) and the cytokine influences several aspects of gliomas [3-5]. Cygb is the fourth member of the vertebrate globin family and was identified independently by three groups shortly thereafter [6]. The functions of Cygb remain to be elucidated; however, it may include detoxification of reactive oxygen species (ROS) and

\footnotetext{
* Correspondence: xzy3175@yahoo.com.cn; Guoj_zhang@yahoo.com 'Department of Neurosurgery, Second Affiliated Hospital of Shantou University Medical College, North Dongxia Rd, Shantou 515041, Guangdong, China

${ }^{7}$ The Breast Center, Cancer Hospital of Shantou University Medical College, Raoping Rd, Shantou 515031, Guangdong, China

Full list of author information is available at the end of the article
}

scavenging NO [7,8]. Although the function of Cygb in vivo remains largely unknown, decreased expression of Cygb and the hypermethylation of the Cygb promoter has been reported in patients with tylosis, non-small-cell lung carcinomas, head and neck cancers, ovarian cancers, and breast cancers [9-12]. Those results suggest that Cygb may function as a tumor suppressor gene [13].

Cygb loss has been reported to be associated with increased cancer cell proliferation, elevated extracellular signal-regulated kinase and Akt activation, overexpression of interleukin-6 (IL-6) [14]. Deregulated signaling through phosphatidylinositol-3 kinase (PI-3K)/Akt pathways has been implicated in the malignant transformation of glial cells [15]. Akt is known to regulate actin cytoskeleton reorganization that plays role in tumor cell migration and invasion [16], and inhibition of Akt prevents glioma cell growth [17]. IL-6 is implicated as major regulators of

\section{Biomed Central}

(C) 2013 Xu et al.; licensee BioMed Central Ltd. This is an Open Access article distributed under the terms of the Creative Commons Attribution License (http://creativecommons.org/licenses/by/2.0), which permits unrestricted use, distribution, and reproduction in any medium, provided the original work is properly cited. 
glioma cell growth and invasiveness. IL-6 regulates the immune response, preferentially activates the signal transducer and activator of transcription-3 (STAT-3), leading to dimerization, nuclear translocation and binding to IFN-c -activated site-like DNA elements [18]. IL-6 cytokine has been extensively studied in astroglial tumors at the mRNA [19] and protein level $[20,21]$ and has been proposed as a determinant of brain tumor progression [22]. It has been shown in experimental models that development of glioblastoma requires the presence of IL-6 [23]. Knowing that IL-6 functions as a downstream mediator for tumor necrosis factor- $\alpha$ (TNF- $\alpha$ ) [24], IL-6 is also recognized as potent regulators of angiogenesis [vascular endothelial growth factor (VEGF)] [25]. However, in gliomas, few previous studies have focused on the correlation between Cygb and VEGF. The relationship between Cygb and production of immunosuppressive cytokines (IL-6, TNF $\alpha$, et al) by tumor cells in gliomas also needs to be confirmed. The paucity of prognostic information regarding Cygb expression and the prognostic role of PI3K/Akt signaling in gliomas prompted us to undertake the present study.

In the clinical setting, the histological grading is a key factor for predicting the biological behavior of gliomas and influencing the choice of therapies, particularly determining the use of adjuvant radiation and specific chemotherapy protocols [26,27]. However, to our knowledge, the exact relationship between histological grading and Cygb expression in tumor cells of glioma has not yet been elucidated. In addition, histological grading makes a contribution toward an estimate of recurrence in gliomas, while a possible relationship between Cygb and glioma recurrence remains to be confirmed.

Therefore, the first goal of this study was to determine Cygb, PI3K, phosphorylated (p)-Akt, IL-6, TNF $\alpha$ and VEGF expression in gliomas. And the second goal of this study was to examine the interaction between CygbPI3K/Akt signaling and cytokines (IL-6, TNF $\alpha$, VEGF), to assess possible relationships of these molecules with clinicopathological features and patients' survival.

\section{Methods}

\section{Patient's description}

The study was carried out in patients with histologically confirmed high and low grade gliomas operated on in the Department of Neurosurgery of Second Affiliated Hospital of Shantou University Medical College between January of 2002 and December of 2011. 88 patients were selected according to our inclusion criteria, which were as follows: All patients with intracranial gliomas for whom archival primary tumor material at diagnosis, age over 18 years old, no previous history of any tumor, no administration of antiepileptic drugs or steroids for more than 3 days, no chemotherapy or radiotherapy received before surgery. Histological sections of the resected primary specimens were reviewed by a senior pathologist according to the criteria of WHO histological classification [28]. All the enrolled patients had received brain tumor resection. After surgery, the patients with glioblastoma multiforme and anaplastic astrocytoma (WHO grade IV and III) were treated with teniposide $\left(70 \mathrm{mg} / \mathrm{m}^{2} /\right.$ day, 3 consecutive days during each 42-day cycle) for 4-6 cycles. The patients with astrocytoma (WHO grade II) were treated with teniposide for 1 cycle only after the initial surgery. The patients with oligodendrogliomas and oligoastrocytoma underwent PCV chemotherapy [procarbazine, methyl-1-(2-chloroethyl)-1-nitrosourea (CCNU), and vincristine] every 6 weeks (42-day cycles) for 2-5 cycles [29]. In the study, written informed consents were obtained for all patients, and the study was approved by the Medical Ethics Committee of the Second Affiliated Hospital Shantou University Medical College.

\section{Immunohistochemical staining and scoring}

Three continual sections of $4-5 \mu \mathrm{m}$ sections were subjected to immunostaining using a SP Kit (DAKO, Denmark). Slides were deparaffinized in xylene and rehydrated in decreasing concentrations of ethanol and rinsed in phosphate-buffered saline. The slides were incubated with hydrogen peroxide for 20 min following microwave heating with $10 \mathrm{mM}$ citrate buffer $(\mathrm{pH} 6.0$; Sigma-Aldrich, Germany) at 2-min intervals for a total of $10 \mathrm{~min}$. After blocking with normal serum for $30 \mathrm{~min}$, the slides were incubated with rabbit polyclonal antibody. The following antibodies were used: anti-Cygb diluted 1:300(bs-0590R, Bioss), anti-PI3K diluted 1:200(bs0128R, Bioss), anti-Akt diluted 1:200(bs-0115R, Bioss), anti-IL-6 diluted 1:300(bs-0781R, Bioss), anti-TNF $\alpha$ diluted 1:300(bs-0078R, Bioss), and anti-VEGF diluted 1:200(RAB-0157, Maixin_Bio). In addition, all cases had been stained CD34 for microvessel counting using antiCD34 antibody diluted 1:100(bs-2038R, Bioss). All rabbit polyclonal antibodies used were provided by Biosynthesis (Beijing, China). The incubation time was $1 \mathrm{~h}$ at room temperature for $\mathrm{CD} 34$ and $20 \mathrm{~h}$ at $4^{\circ} \mathrm{C}$ for Cygb, PI3K, p-Akt, IL-6, TNF $\alpha$ and VEGF. Slides were detected by SP Kit for $30 \mathrm{~min}$ at room temperature and followed by developing with diaminobenzidine for visualization. Negative controls included sections where primary antibody had been substituted by nonimmune serum.

Cygb, PI3K, p-Akt, IL-6, TNF $\alpha$ and VEGF immunoreactivity was evaluated by light microscopy by two experienced pathologists without knowledge of the clinical information. If a discrepancy occurred between the assessments of the two observers, the slides were reassessed in a combined session without information of the previous scores. In each section, the percentage of tumor cells with Cygb, PI3K, p-Akt, IL-6, TNF $\alpha$ and 
VEGF immunoreactivity was calculated in at least 500 cells counted in several randomly chosen high power fields. In each case, the percentage of tumor cells with Cygb, PI3K, p-Akt, IL-6, TNF $\alpha$ and VEGF immunoreactivity was the mean value of the 3 continual sections. High expression of proteins was more than median value of tumor cells with positive staining, whereas low expression was less than median value. Intratumoral microvessel density (IMD) was observed in areas of most intense neovascularization or hotspots in the tumor by light microscopy. After the area of the highest neovascularization was determined, single microvessels were manually counted on $\mathrm{a} \times 200$ field by two independent observers without knowledge of the patient outcome. Any brownstained endothelial cell or cell cluster that was clearly separated from adjacent microvessels was considered as a single, countable microvessel, and the IMD value of each sample was the mean of the independent microvessels counts by two observers.

\section{Statistical analysis}

All statistical analysis was carried out by SPSS 17.0 software for Windows. In the basic statistical analysis Cygb, PI3K, p-Akt, IL-6, TNF $\alpha$ and VEGF expressions were treated as continuous variables to avoid any "datadriven" categorization. Associations of Cygb, PI3K, pAkt, IL-6, TNF $\alpha$ and VEGF expression with clinicopathological characteristics were tested using non-parametric tests with correction for multiple comparisons (KruskalWallis ANOVA, Mann-Whitney U-test and Spearman's rank correlation coefficient). Correlations among Cygb, PI3K, p-Akt, IL-6, TNF $\alpha$ and VEGF and microvascular parameters were tested with Spearman's correlation coefficient. Vascular density was given as the mean \pm SD as indicated. Data were analyzed by one-way ANOVA with Dunnett's post hoc test and Turkey's post hoc test for multigroup comparisons. The survival curve of patients was determined by the Kaplan-Meier method and Cox regression, and statistical evaluation was performed using the log rank test. All results with a two-sided $p$ level $<0.05$ were considered statistically significant.

\section{Results}

\section{Patients characteristics}

The patients were 51 males and 37 females with median age of 41 years old (range 18-74). The high grade group included 15 patients with glioblastoma multiforme (GBM), (WHO grade IV), 26 patients with anaplastic gliomas (AG), (WHO grade III) and 47 patients with low grade gliomas (LGG), (WHO grade I-II). The followedup was made by telephone call or clinic with median of 20 months (3-80 months). 43 disease-specific deaths were recorded during follow-up.
Immunohistochemical assessment of Cygb, PI3K, p-Akt, IL-6, TNFa and VEGF in gliomas

Cytoplasmic and nucleus surface positive staining for Cygb, PI3K and p-Akt was observed in tumor cells of gliomas, no positive staining was shown in tumor cells of negative controls (Figure 1). Immunostaining signal of IL-6, TNF $\alpha$ and VEGF was localized in the cytoplasm of tumor cells (Figure 2) and CD34 was localized in endothelial cells of newly formed vessels. On serial section, CYGB, PI3K, p-Akt, IL-6, TNF $\alpha$ and VEGF could be detected in the same area of tumor cells, at least in a part of them.

Positive staining for Cygb, PI3K, p-Akt, IL-6, TNF $\alpha$ and VEGF were observed in 10\%-86\% (median value: 39\%), 3\%-62\% (median value: 20\%), 5\%-67\% (median value: $21 \%$ ), $4 \%-70 \%$ (median value: $35 \%$ ), 3\%-67\% (median value: $30 \%$ ) and $10 \%-89 \%$ (median value: $56 \%$ ), respectively. Low expression of Cygb was significantly associated with the higher histological grading and tumor recurrence. High expression of PI3K, p-Akt, IL-6, TNF $\alpha$ and VEGF were significantly associated with the higher histological grade, and high expression of PI3K, p-Akt and IL-6 were significantly associated with tumor recurrence. There was no correlation observed between Cygb, PI3K, p-Akt, IL-6, TNF $\alpha$ or VEGF expression and age of patients (Table 1). However, Cygb expression was significantly higher in female patients.

\section{Correlations between Cygb, PI3K, p-Akt, IL-6, TNFa, and VEGF immunoreactivity in gliomas}

A significant negative correlation emerged between Cygb expression and PI3K or p-Akt expression $(r=-0.728$, $\mathrm{p}<0.0001$ and $\mathrm{r}=-0.711, \mathrm{p}<0.0001$ respectively). High PI3K and p-Akt expression was correlated with high IL-6 $(\mathrm{r}=0.302, \mathrm{p}=0.004$ and $\mathrm{r}=0.328, \mathrm{p}=0.002$, respectively) and TNF $\alpha$ expression $(r=0.278, p=0.009$ and $r=0.308$, $\mathrm{p}=0.004$, respectively). IL-6 expression levels were positively associated with TNF $\alpha$ expression ( $\mathrm{r}=0.724$, $\mathrm{p}=<0.0001)$. There was significant negative correlation observed between Cygb expression and IL-6 or TNF $\alpha$ or VEGF expression $(\mathrm{r}=-0.370, \mathrm{p}<0.0001, \mathrm{r}=-0.345$, $\mathrm{p}=0.001$ and $\mathrm{r}=-0.378, \mathrm{p}<0.0001$ respectively). High expression of IL- 6 and TNF $\alpha$ exhibited a close correlation with high expression of VEGF in the tumor cells $(\mathrm{r}=0.714, \mathrm{p}<0.0001$ and $\mathrm{r}=0.702, \mathrm{p}<0.0001$ respectively) (Table 2).

\section{Correlation of Cygb, PI3K, p-Akt, IL-6, TNFa and VEGF expression with IMD in gliomas}

Microvessels in gliomas, specifically stained by antiCD34 immunostaining, were observed in all specimens, and scored as IMD. The mean IMD value was $30 / \mathrm{HPF}$, but with great individual variation (range 12-56). The correlation between IMD and proteins expression in 


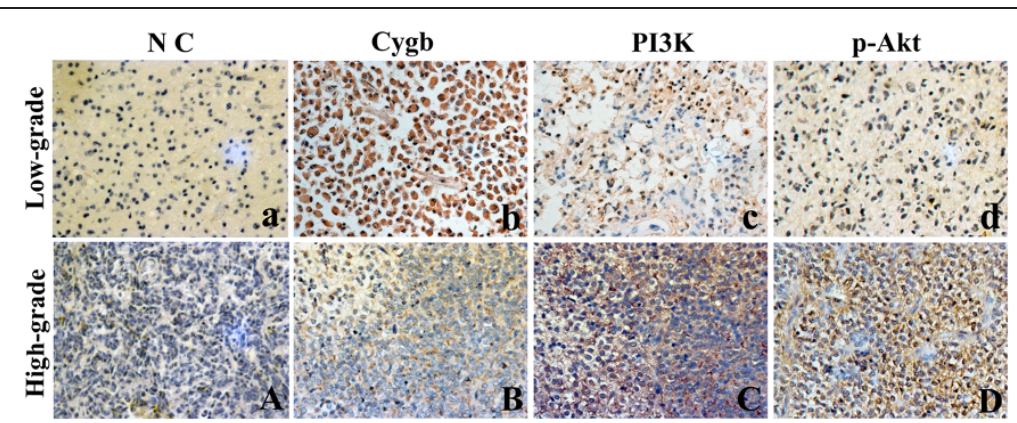

Figure 1 Immunohistochemical staining of Cygb, PI3K and p-Akt in low and high grade of glioma tissues. Negative control (NC) of low (a) and high (A) grade gliomas showed no positive staining cells. Strong and diffused expression of Cygb was found in low-grade gliomas (b); in high-grade gliomas, positive staining of Cygb was shown focally and weakly (B). Low-grade gliomas showed low expression of PI3K (c) and p-Akt (d) positive tumor cells in serial section. High expression of PI3K (C) and p-Akt (D) was shown in high-grade gliomas. (magnification: $\times 400)$.

gliomas is shown in Table 2. Cygb expression was negatively correlated with IMD. There was a positive correlation between PI3K, p-Akt, IL-6, TNF $\alpha$ and VEGF expression with IMD. The IMD was significantly higher in tumors with high expression of PI3K, p-Akt, IL-6, TNF $\alpha$ or VEGF than in tumors with lower protein expression (Table 2).

\section{Association of Cygb, PI3K, p-Akt, IL-6, TNFa and VEGF expression with survival of patients with gliomas}

The 88 cases were followed up from 3 to 80 months with a mean period of 20 months, and 43 patients (48.9\%) had died of their tumor during this period. The mean survival time of patients with high-grade tumors (WHO III-IV) and low-grade tumors (WHO I-II) were $17.3 \pm 1.7$ and $60.7 \pm 4.2$ months, respectively; there was a statistically significant difference $(\mathrm{p}<0.01)$.

Univariate survival analysis (Kaplan-Meier analysis) was carried out in all cases. Figure 3 showed KaplanMeier curves of Cygb, PI3K, p-Akt, IL-6, TNF $\alpha$ and VEGF expression with overall survival in the entire case. The parameters adversely affecting survival in all cases

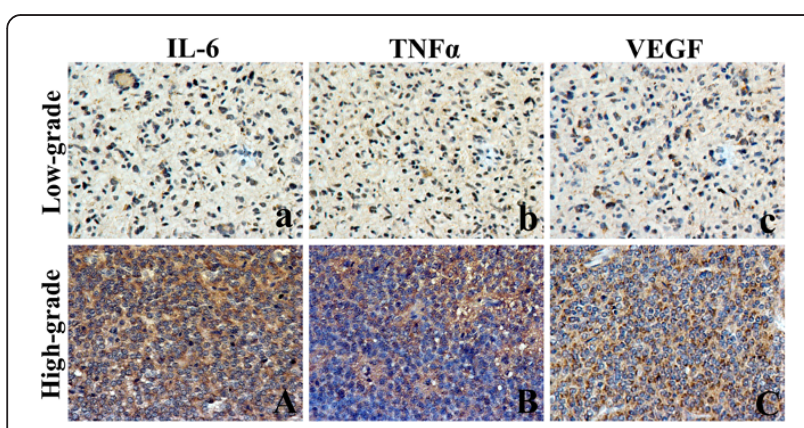

Figure 2 Immunohistochemical staining of IL-6, TNFa and VEGF in low and high grade of glioma tissues. Low-grade gliomas showed low expression of IL-6 (a), TNFa (b) and VEGF (c) positive tumor cells in serial section. High expression of IL-6 (A), TNFa (B) and VEGF $(\mathbf{C})$ was shown in high-grade gliomas.

(magnification: $\times 400$ ) were high histologic grade $(\mathrm{p}<0.01)$, tumor recurrence $(\mathrm{p}<0.01)$, decreased Cygb expression $(\mathrm{p}<0.01)$, increased PI3K expression $(\mathrm{p}<0.01)$, increased $\mathrm{p}$-Akt expression ( $\mathrm{p}$ $<0.01)$ and increased VEGF expression $(\mathrm{p}=0.023)$ (Table 3). The overall survival is $42.9 \pm 3.6$ months for all patients. The median survival time was $62.4 \pm 4.8$ months for patients overexpressing Cygb compared to $23.8 \pm 3.1$ months for patients with lower expression. The median survival time for patients whose tumors displayed overexpression of PI3K and p-Akt were $29.2 \pm$ 4.2 months and $29.6 \pm 4.3$ months respectively. The corresponding figure for patients whose tumors presented increased expression of IL-6, TNF $\alpha$ and VEGF were $40.1 \pm 5.0$ months, $44.2 \pm 5.3$ and $34.0 \pm 3.6$ months respectively (Table 3 ).

Multivariate survival analysis (Cox regression model) results including all parameters for the 88 patients, for whom Cygb, PI3K, p-Akt, IL-6, TNF $\alpha$, VEGF and CD34 staining results were available are presented in Table 4. Only histological grade and Cygb expression appeared to affect survival in all cases (Table 4). Other parameters, such as age, gender, tumor recurrence, angiogenesis and expression of PI3K, p-Akt, IL-6, TNF $\alpha$ and VEGF did not show any association with the survival of the patients (Table 4).

\section{Discussion}

Cygb as a tumor suppressor gene has been demonstrated in hepatocellular carcinoma, lung cancer and breast cancer [9-12]. Expression of Cygb has been reported in various human tumors, including gliomas [5]. This study showed that Cygb expression was found to inversely associate with higher histological grade in gliomas, and lower expression of Cygb is closely related to a shorter survival time of patients using either univariate or multivariate analysis. These results indicated that Cygb may function not only as a tumor suppressor gene, which was supported by previous studies, but also as a prognostic factor. Previous studies have indicated that Cygb 
Table 1 Correlation among Cygb, VEGF, PI3K, p-Akt, IL-6,TNFa expression and clinicopathological parameters of patients with gliomas

\begin{tabular}{|c|c|c|c|c|c|c|c|c|c|}
\hline \multirow[t]{2}{*}{ Vareable } & \multirow[t]{2}{*}{ Age $(n=88)$} & \multicolumn{2}{|c|}{ Gender } & \multicolumn{4}{|c|}{ Histological grade } & \multicolumn{2}{|c|}{ Recurrence } \\
\hline & & $\begin{array}{c}\text { Male } \\
(n=51)\end{array}$ & $\begin{array}{l}\text { Female } \\
(n=37)\end{array}$ & $\begin{array}{l}\text { Grade I } \\
(n=10)\end{array}$ & $\begin{array}{l}\text { Grade II } \\
(n=37)\end{array}$ & $\begin{array}{c}\text { Grade III } \\
(n=26)\end{array}$ & $\begin{array}{c}\text { Grade IV } \\
(n=15)\end{array}$ & No $(n=31)$ & Yes $(n=57)$ \\
\hline \multicolumn{10}{|c|}{ Cygb expression } \\
\hline Median & $39 \%$ & $30 \%$ & $52 \%$ & $56.5 \%$ & $66 \%$ & $31.5 \%$ & $14 \%$ & $64 \%$ & $22 \%$ \\
\hline Range & $10 \%-86 \%$ & $11 \%-85 \%$ & $10 \%-86 \%$ & $20 \%-86 \%$ & $12 \%-85 \%$ & $18 \%-52 \%$ & $10 \%-20 \%$ & $25 \%-86 \%$ & $10 \%-85 \%$ \\
\hline$p$ value & $0.439^{\star}(r=-0.083)$ & $0.031^{\triangle}$ & & $<0.01^{\bullet}$ & & & & $<0.01^{\Delta}$ & \\
\hline \multicolumn{10}{|c|}{ PI3K expression } \\
\hline Median & $20 \%$ & $30 \%$ & $15 \%$ & $13 \%$ & $11 \%$ & $32.5 \%$ & $38 \%$ & $12 \%$ & $27 \%$ \\
\hline Range & $3 \%-62 \%$ & $3 \%-62 \%$ & $3 \%-60 \%$ & $3 \%-37 \%$ & $3 \%-58 \%$ & $7 \%-60 \%$ & $24 \%-62 \%$ & $3 \%-56 \%$ & $5 \%-62 \%$ \\
\hline$p$ value & $0.236^{\star}(r=0.128)$ & $0.055^{\Delta}$ & & $<0.01^{\star}$ & & & & $<0.01^{\triangle}$ & \\
\hline \multicolumn{10}{|c|}{ p-Akt expression } \\
\hline Median & $21 \%$ & $27 \%$ & $17 \%$ & $15 \%$ & $14 \%$ & $31.5 \%$ & $40 \%$ & $15 \%$ & $29 \%$ \\
\hline Range & $5 \%-67 \%$ & $5 \%-62 \%$ & $5 \%-67 \%$ & $5 \%-38 \%$ & $5 \%-60 \%$ & $10 \%-57 \%$ & $27 \%-67 \%$ & $5 \%-58 \%$ & $7 \%-67 \%$ \\
\hline$p$ value & $0.135 \bullet(r=0.161)$ & $0.091^{\triangle}$ & & $<0.01^{\star}$ & & & & $<0.01^{\Delta}$ & \\
\hline \multicolumn{10}{|c|}{ IL-6 expression } \\
\hline Median & $35 \%$ & $35 \%$ & $35 \%$ & $22.5 \%$ & $27 \%$ & $30 \%$ & $46 \%$ & $25 \%$ & $40 \%$ \\
\hline Range & $4 \%-70 \%$ & $5 \%-70 \%$ & $4 \%-68 \%$ & $4 \%-50 \%$ & $7 \%-68 \%$ & $8 \%-70 \%$ & $12 \%-66 \%$ & $4 \%-68 \%$ & $5 \%-70 \%$ \\
\hline$p$ value & $0.859^{\star}(r=0.019)$ & $0.472^{\Delta}$ & & $0.019^{\star}$ & & & & $0.041^{\Delta}$ & \\
\hline \multicolumn{10}{|c|}{ TNFa expression } \\
\hline Median & $30 \%$ & $27 \%$ & $33 \%$ & $16 \%$ & $22 \%$ & $33 \%$ & $47 \%$ & $21 \%$ & $35 \%$ \\
\hline Range & $3 \%-67 \%$ & $3 \%-67 \%$ & $4 \%-67 \%$ & $4 \%-46 \%$ & $3 \%-62 \%$ & $3 \%-67 \%$ & $10 \%-62 \%$ & $3 \%-62 \%$ & $3 \%-67 \%$ \\
\hline$p$ value & $0.857^{\star}(r=-0.019)$ & $0.543^{\Delta}$ & & $0.010^{\star}$ & & & & $0.123^{\Delta}$ & \\
\hline \multicolumn{10}{|c|}{ VEGF expression } \\
\hline Median & $56 \%$ & $55 \%$ & $58 \%$ & $44 \%$ & $32 \%$ & $60.5 \%$ & $67 \%$ & $30 \%$ & $61 \%$ \\
\hline Range & 10\%-89\% & $10 \%-87 \%$ & $11 \%-89 \%$ & 11\%-78\% & $10 \%-85 \%$ & $10 \%-87 \%$ & $12 \%-89 \%$ & $10 \%-85 \%$ & $10 \%-89 \%$ \\
\hline$p$ value & $0.178^{\circ}(r=0.145)$ & $0.337^{\Delta}$ & & $0.027^{\star}$ & & & & $0.061^{\triangle}$ & \\
\hline
\end{tabular}

- Results Spearman's correlation coefficient. $\triangle$ Results Mann-Whitney U test. $\bullet$ Results Kruskal-Wallis ANOVA.

loss was associated with increased cancer cell proliferation and overexpression of IL-1, IL-6, VEGF, TNFo, and TNFb mRNAs in cancer development in the liver and lungs of mice exposed to N,N-diethylnitrosamine [14]. As we known, IL-1, IL-6, VEGF and TNF $\alpha$ are immunosuppressive cytokines. Immunosuppressive cytokines and tumor biology are closely intertwined since increased production of immunosuppressive cytokines in cancer cells not only had a stimulating effect on tumor cell growth and proliferation but also regarded as an indispensable participation in tumor progression were known to evade immune surveillance [30,31]. We postulated that Cygb was likely to influence the prognosis of glioma patients by effect on production of immunosuppressive cytokines and angiogenesis in gliomas.

In the present study, we found a direct relationship between Cygb expression level and tumor recurrence, independent of vascular density and angiogenic factor expression. In particular, Grade I-II gliomas with lower
Cygb expression were found more likely to recur during the period of follow-up despite the low level of proliferative activity in this tumor. In the clinic, neither histopathologic nor clinical data were currently taken as reliable recurrence predictors for low-grade gliomas, especially for grade I tumors. Although the number of glioma samples examined in this study was not sufficient to allow us to draw a definite conclusion, our findings indicated that Cygb expression level could be used as a predictor for the recurrence of gliomas. There are some histological features of gliomas associated with grade and prognosis, such as the extent of vascular density [32]. In our study, we found that low expression of Cygb in glioma cells was closely associated with higher microvessel density in tumor, which suggested Cygb might contribute partly to angiogenesis of gliomas. These results indicated that Cygb loss in tumor cells might play an important role in tumor progression through the induction of angiogenesis. In the present 
Table 2 Spearman's correlation coefficient between Cygb, PI3K, p-Akt, IL-6, TNFa, VEGF expression and IMD value

\begin{tabular}{ccccccc}
\hline & Cygb & PI3K & p-Akt & IL-6 & TNFa & VEGF \\
\hline PI3K & & & & & & \\
$\mathbf{r}$ & -0.728 & & & & & \\
$\mathbf{p}$ & $<0.0001$ & & & & & \\
$\mathbf{p - A k t}$ & & & & & & \\
$\mathbf{r}$ & -0.711 & 0.818 & & & & \\
$\mathbf{p}$ & $<0.0001$ & $<0.0001$ & & & & \\
IL-6 & & & & & & \\
$\mathbf{r}$ & -0.370 & 0.302 & 0.328 & & & \\
$\mathbf{p}$ & $<0.0001$ & 0.004 & 0.002 & & & \\
TNFa & & & & & & \\
$\mathbf{r}$ & -0.345 & 0.278 & 0.308 & 0.724 & & \\
$\mathbf{p}$ & 0.001 & 0.009 & 0.004 & $<0.0001$ & & \\
VEGF & & & & & & \\
$\mathbf{r}$ & -0.378 & 0.395 & 0.406 & 0.714 & 0.702 & \\
$\mathbf{p}$ & $<0.0001$ & $<0.0001$ & $<0.0001$ & $<0.0001$ & $<0.0001$ & \\
IMD & & & & & & \\
$\mathbf{r}$ & -0.514 & 0.396 & 0.426 & 0.710 & 0.691 & 0.0001 \\
$\mathbf{p}$ & $<0.0001$ & $<0.0001$ & $<0.0001$ & $<0.0001$ & $<0.0001$ & $<0.001$ \\
\hline & & & & & & \\
\end{tabular}

study, we found that IL-6 high expression in gliomas was significantly correlated with low expression of Cygb, as well as higher histological grade and increased neovascularization in tumors.

Previous study found that PI3K/Akt pathway is deregulated in GBM [33] and activation of this pathway has been shown to be associated with reduced patient survival [34]. In our study, we found low level of Cygb expression was associated with an increased Akt and PI3K expression in gliomas. The increased levels of Akt and PI3K correlated with a marked elevation of IL- 6 and TNFo. These data suggested that low expression of Cygb, together with high expression of IL-6, TNF $\alpha$, Akt and PI3K might play an important role in the development of glioma.

In this particular cohort, $62.2 \%$ (23 out of 37 ) of female patients was with low grade tumors while about $47 \%$ (24 out of 51 ) of male patients was diagnosed as low grade tumors. The selection bias was found to cause the gender difference in terms of histological grade. Thus, the finding that low expression of Cygb was significantly associated with female gender was most likely because of difference in histological grade.

In further study, we will investigate the role of Cygb expression by overexpressing via introducing exogenous

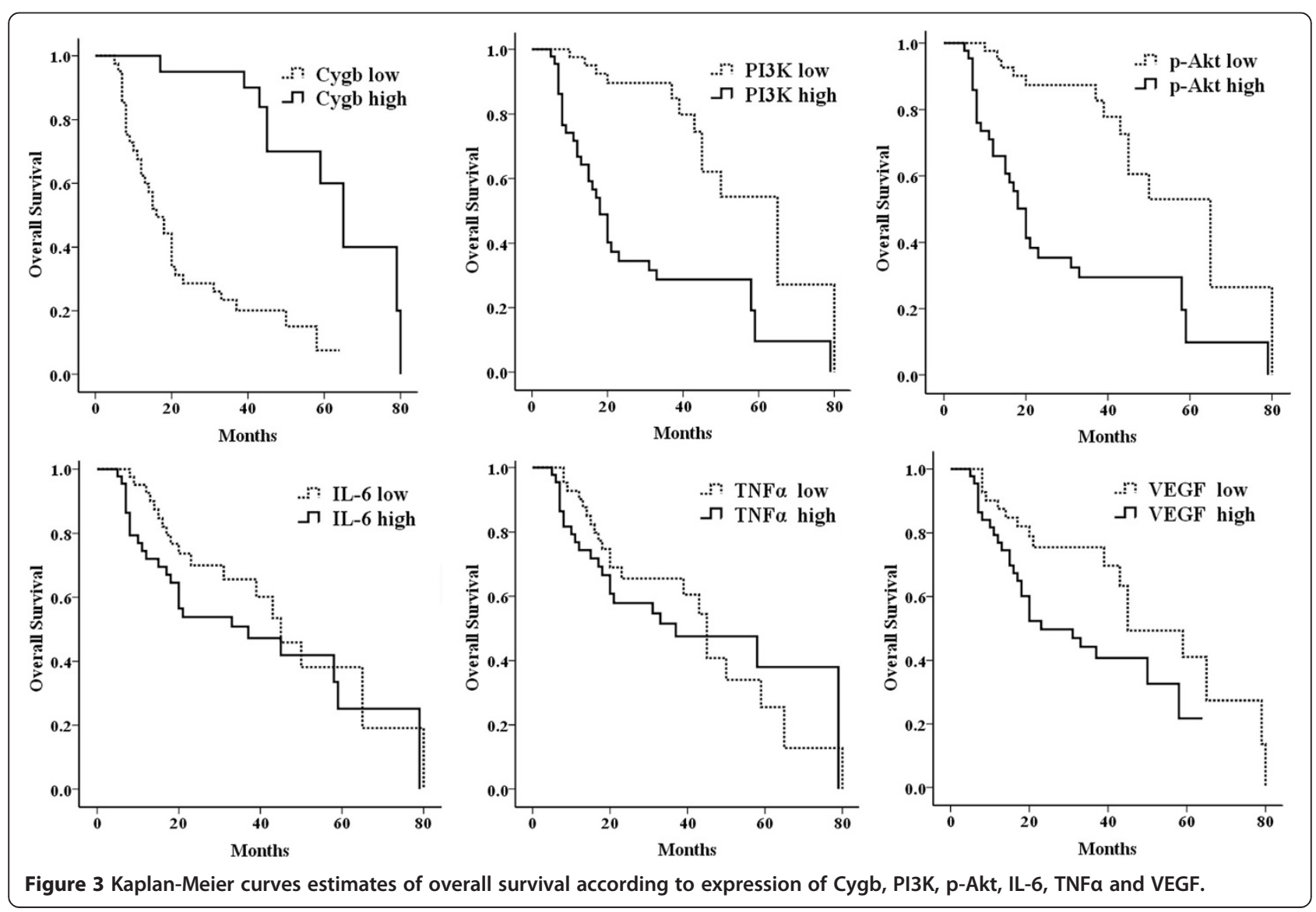


Table 3 Kaplan-Meier analysis for overall survival rate of patients with gliomas

\begin{tabular}{|c|c|c|c|}
\hline Characteristics & Mean survival time $\pm \mathrm{SE}$ & 95\% Confidence interval (months) & $P$ values \\
\hline \multicolumn{4}{|l|}{ Age (years) } \\
\hline$<41$ & $49.8 \pm 5.4$ & $39.2-60.4$ & 0.055 \\
\hline$\geq 41$ & $35.7 \pm 4.5$ & $26.8-44.5$ & \\
\hline \multicolumn{4}{|l|}{ Gender } \\
\hline Male & $39.2 \pm 4.6$ & $30.1-48.2$ & 0.086 \\
\hline Female & $46.2 \pm 4.3$ & $37.8-54.6$ & \\
\hline \multicolumn{4}{|l|}{ Histological grade } \\
\hline Low-grade (WHOI-II) & $60.7 \pm 4.2$ & $52.5-69.0$ & $<0.01$ \\
\hline High-grade (WHO III-IV) & $17.3 \pm 1.7$ & $14.0-20.7$ & \\
\hline \multicolumn{4}{|l|}{ Tumor recurrence } \\
\hline No & $61.8 \pm 1.1$ & $59.6-63.9$ & $<0.01$ \\
\hline Yes & $33.1 \pm 3.7$ & $25.9-40.3$ & \\
\hline \multicolumn{4}{|l|}{ Cygb expression } \\
\hline Low expression $(<39 \%)$ & $23.8 \pm 3.1$ & $17.8-29.8$ & $<0.01$ \\
\hline High expression ( $\geq 39 \%$ ) & $62.4 \pm 4.8$ & $53.0-71.7$ & \\
\hline \multicolumn{4}{|l|}{ PI3K expression } \\
\hline Low expression $(<20 \%)$ & $56.5 \pm 5.1$ & $46.4-66.5$ & $<0.01$ \\
\hline High expression $(\geqq \%)$ & $29.2 \pm 4.2$ & 20.9-37.5 & \\
\hline \multicolumn{4}{|l|}{ p-Akt expression } \\
\hline Low expression $(<21 \%)$ & $55.4 \pm 5.1$ & $45.3-65.4$ & $<0.01$ \\
\hline High expression ( $\geqq \mathbf{2 0} \%$ ) & $29.6 \pm 4.3$ & $21.2-38.1$ & \\
\hline \multicolumn{4}{|l|}{ IL-6 expression } \\
\hline Low expression(<35\%) & $45.9 \pm 5.2$ & $35.7-56.2$ & 0.157 \\
\hline High expression ( $\geqq 35 \%)$ & $40.1 \pm 5.0$ & $30.3-49.8$ & \\
\hline \multicolumn{4}{|l|}{ TNFa expression } \\
\hline Low expression $(<30 \%)$ & $62.9 \pm 4.6$ & $33.8-51.9$ & 0.569 \\
\hline High expression $(\geqq 30 \%)$ & $44.2 \pm 5.3$ & $33.7-54.6$ & \\
\hline \multicolumn{4}{|l|}{ VEGF expression } \\
\hline Low expression $(<56 \%)$ & $50.1 \pm 5.2$ & $39.9-60.4$ & 0.023 \\
\hline High expression $(\geqq 56 \%)$ & $34.0 \pm 3.6$ & $26.9-41.2$ & \\
\hline
\end{tabular}

Table 4 Cox regression model for multivariate analyses of prognostic factor in gliomas

\begin{tabular}{lllll}
\hline Variable & Wald & Hazard ratio & 95\% Confidence interval & P value \\
\hline Age (>41 vs <41) & 0.678 & 0.722 & $0.333-1.567$ & 0.410 \\
Gender (male vs. female) & 0.830 & 0.696 & $0.319-1.518$ & 0.362 \\
Histological grade (low-grade vs. high-grade) & 14.358 & 15.320 & $3.734-62.857$ & $<0.01$ \\
Tumor recurrence (no vs. yes) & 3.142 & 6.383 & $0.822-49.563$ & 0.076 \\
Cygb expression (low vs. high) & 5.254 & 0.235 & $0.068-0.811$ & 0.022 \\
PI3K expression (low vs. high) & 0.808 & 3.161 & $0.275-38.859$ & 0.369 \\
p-Akt expression (low vs. high) & 0.323 & 0.503 & $0.047-5.374$ & 0.570 \\
IL-6 expression (low vs. high) & 2.137 & 2.730 & $0.710-10.493$ & 0.144 \\
TNFa expression (low vs. high) & 1.818 & 0.390 & $0.099-1.532$ & 0.178 \\
VEGF expression (low vs. high) & 1.119 & 0.560 & $0.191-1.641$ & 0.290 \\
Angiogenesis (IMD value) & 0.375 & 1.016 & $0.966-1.068$ & 0.540 \\
\hline
\end{tabular}


Cygb or silencing Cygb protein via siRNA in cultured glioma cells. Moreover, we will try to develop animal models of gliomas either by transgenic models or by subcutaneous injection to charcterize the significance of Cygb overexpression or inhibition.

\section{Conclusion}

In conclusion, the present data indicated prognostic significance of Cygb in gliomas: correlation with PI3K, Akt, IL-6, TNF $\alpha$, VEGF, microvessel morphometry and survival of patients with gliomas. Cygb loss may play an important role in contributing to production of immunosuppressive cytokines and angiogenesis in gliomas. Histological grade and Cygb expression in tumors are independent predictors for the prognosis of patients with gliomas. Moreover, Cygb expression level may help determine whether aggressive therapy is necessary, particularly for those gliomas with lower WHO grades.

\begin{abstract}
Abbreviations
Cygb: Cytoglobin; PI3K: Phosphatidylinositol-3 kinase; IL-6: Interleukin-6; TNFa: Tumor necrosis factor- $a$; VEGF: Vascular endothelial growth factor; IMD: Intratumoral microvessel density; ROS: Reactive oxygen species; STAT3: Signal transducer and activator of transcription-3; DAB: Diaminobenzidine.
\end{abstract}

\section{Competing interests}

The authors declare that they have no competing interests.

\section{Authors' contributions}

HWX and YJH are co-first authors, and they made equal contributions to this work. HWX performed immunohistochemical staining, data analysis and drafted the manuscript. YJH followed up the cases and carried out the statistical analysis. LL, YCG, ZRZ, XPL, WZ and ML participated in collecting correlative cases. $\mathrm{HHH}$ and $\mathrm{XLW}$ reviewed the histological sections of the cases. GJZ and ZYX are co-corresponding authors. GJZ conceived of the study and participated in its design. ZYX and KM participated in the overall design. All authors read and approved the final manuscript.

\section{Acknowledgements}

This work was supported by: State Key Development Program for Basic Research of China (No. 2011CB707705) and Medical Scientific Research Foundation of Guangdong Province, China (No. A2012396).

\section{Author details}

${ }^{1}$ Department of Neurosurgery, Second Affiliated Hospital of Shantou University Medical College, North Dongxia Rd, Shantou 515041, Guangdong, China. ${ }^{2}$ Research Center for Translational Medicine, Second Affiliated Hospital of Shantou University Medical College, North Dongxia Rd, Shantou 515041, Guangdong, China. ${ }^{3}$ Department of pediatrics, Second Affiliated Hospital of Shantou University Medical College, North Dongxia Rd, Shantou 515041, Guangdong, China. ${ }^{4}$ Department of pathology, Second Affiliated Hospital of Shantou University Medical College, North Dongxia Rd, Shantou 515041, Guangdong, China. ${ }^{5}$ Department of pathology, Cancer Hospital of Shantou University Medical College, Raoping Rd, Shantou 515031, Guangdong, China. ${ }^{6}$ Department of Surgery and Centre for Cancer Research, LKS Faculty of Medicine, The University of Hong Kong, Pokfulam, Hong Kong, China. ${ }^{7}$ The Breast Center, Cancer Hospital of Shantou University Medical College, Raoping Rd, Shantou 515031, Guangdong, China.

Received: 6 January 2013 Accepted: 16 May 2013

Published: 20 May 2013

\section{References}

1. Louis DN: Molecular pathology of malignant gliomas. Annu Rev Pathol 2006, 1:97-117.
2. Furnari FB, Fenton T, Bachoo RM, Mukasa A, Stommel JM, Stegh A, Hahn WC, Ligon KL, Louis DN, Brennan C, Chin L, DePinho RA, Cavenee WK: Malignant astrocytic glioma: genetics, biology, and paths to treatment. Genes Dev 2007, 21(21):2683-2710.

3. Saidi A, Hagedorn M, Allain N, Verpelli C, Sala C, Bello L, Bikfalvi A, Javerzat S: Combined targeting of interleukin- 6 and vascular endothelial growth factor potently inhibits glioma growth and invasiveness. Int J Cancer 2009, 125(5):1054-1064.

4. Sciume G, Santoni A, Bernardini G: Chemokines and glioma: invasion and more. J Neuroimmunol 2010, 224(1-2):8-12.

5. Fang J, Ma I, Allalunis-Turner J: Knockdown of cytoglobin expression sensitizes human glioma cells to radiation and oxidative stress. Radiat Res 2011, 176(2):198-207.

6. Burmester T, Ebner B, Weich B, Hankeln T: Cytoglobin: a novel globin type ubiquitously expressed in vertebrate tissues. Mol Biol Evol 2002, 19(4):416-421.

7. Fordel E, Thijs L, Martinet W, Schrijvers D, Moens L, Dewilde S: Anoxia or oxygen and glucose deprivation in SH-SY5Y cells: a step closer to the unraveling of neuroglobin and cytoglobin functions. Gene 2007, 398(1-2):114-122.

8. Halligan KE, Jourd'heuil FL, David Jourd'heuil1: Cytoglobin is expressed in the vasculature and regulates cell respiration and proliferation via nitric oxide dioxygenation. J Biol Chem 2009, 284(13):8539-8547.

9. Xinarianos G, McRonald FE, Risk JM, Bowers NL, Nikolaidis G, Field JK, Liloglou T: Frequent genetic and epigenetic abnormalities contribute to the deregulation of cytoglobin in non-small cell lung cancer. Hum Mol Genet 2006, 15(13):2038-2044.

10. Shaw RJ, Hall GL, Woolgar JA, Lowe D, Rogers SN, Field JK, Liloglou T, Risk JM: Quantitative methylation analysis of resection margins and lymph nodes in oral squamous cell carcinoma. Br J Oral Maxillofac Surg 2007, 45(8):617-622.

11. Shaw RJ, Omar MM, Rokadiya S, Kogera FA, Lowe D, Hall GL, Woolgar JA, Homer J, Liloglou T, Field JK, Risk JM: Cytoglobin is upregulated by tumour hypoxia and silenced by promoter hypermethylation in head and neck cancer. Br J Cancer 2009, 101(1):139-144.

12. Gorr TA, Wichmann D, Pilarsky C, Theurillat JP, Fabrizius A, Laufs T, Bauer T, Koslowski M, Horn S, Burmester T, Hankeln T, Kristiansen G: Old proteins new locations: myoglobin, haemoglobin, neuroglobin and cytoglobin in solid tumours and cancer cells. Acta Physiol (Oxf) 2011, 202(3):563-581.

13. Shivapurkar N, Stastny V, Okumura N, Girard L, Xie Y, Prinsen C, Thunnissen FB, Wistuba II, Czerniak B, Frenkel E, Roth JA, Liloglou T, Xinarianos G, Field JK, Minna JD, Gazdar AF: Cytoglobin, the newest member of the globin family, functions as a tumor suppressor gene. Cancer Res 2008, 68(18): 7448-7456.

14. le Thuy T, Morita T, Yoshida K, Wakasa K, lizuka M, Ogawa T, Mori M, Sekiya Y, Momen S, Motoyama H, Ikeda K, Yoshizato K, Kawada N: Promotion of Liver and Lung Tumorigenesis in DEN-Treated Cytoglobin-Deficient Mice. Am J Pathol 2011, 179(2):1050-1060.

15. Rao RD, James CD: Altered molecular pathways in gliomas: an overview of clinically relevant issues. Semin Oncol 2004, 31(5):595-604.

16. Qian Y, Zhong X, Flynn DC, Zheng JZ, Qiao M, Wu C, Dedhar S, Shi X, Jiang $\mathrm{BH}$ : ILK mediates actin filament rearrangements and cell migration and invasion through PI3K/Akt/Rac1 signaling. Oncogene 2005, 24(19): 3154-3165.

17. Gallia GL, Tyler BM, Hann CL, Siu IM, Giranda VL, Vescovi AL, Brem H, Riggins GJ: Inhibition of Akt inhibits growth of glioblastoma and glioblastoma stem-like cells. Mol Cancer Ther 2009, 8(2):386-393.

18. Shuai K, Liu B: Regulation of JAK-STAT signaling in the immune system. Nat Rev Immunol 2003, 3(11):900-911.

19. Tchirkov A, Rolhion C, Bertrand S, Doré JF, Dubost JJ, Verrelle P: IL-6 gene amplification and expression in human glioblastomas. Br J Cancer 2001, 85(4):518-522.

20. Samaras V, Piperi C, Korkolopoulou P, Zisakis A, Levidou G, Themistocleous MS, Boviatsis El, Sakas DE, Lea RW, Kalofoutis A, Patsouris E: Application of the ELISPOT method for comparative analysis of interleukin (IL)- 6 and IL-10 secretion in peripheral blood of patients with astroglial tumours. Mol Cell Biochem 2007, 304(1-2):343-351.

21. Zisakis A, Piperi C, Themistocleous MS, Korkolopoulou P, Boviatsis El, Sakas $D E$, Patsouris E, Lea RW, Kalofoutis A: Comparative analysis of peripheral and localised cytokine secretion in glioblastoma patients. Cytokine 2007, 39(2):99-105. 
22. Tchirkov $A$, Khalil $T$, Chautard $E$, Mokhtari $K$, Véronèse $L$, Irthum $B$, Vago $P$, Kémény $J L$, Verrelle P: Interleukin-6 gene amplification and shortened survival in glioblastoma patients. Br J Cancer 2007, 96(3):474-476.

23. Brantley EC, Benveniste EN: Signal transducer and activator of transcription-3: a molecular hub for signaling pathways in gliomas. Mol Cancer Res 2008, 6(5):675-684.

24. Kamimura D, Ishihara K, Hirano T: IL-6 signal transduction and its physiological roles: the signal orchestration model. Rev Physiol Biochem Pharmacol 2003, 149:1-38.

25. Koch AE, Polverini PJ, Kunkel SL, Harlow LA, DiPietro LA, Elner VM, Elner SG, Strieter RM: Interleukin-8 as a macrophage derived mediator of angiogenesis. Science 1992, 258(5089):1798-1801.

26. Nieder C, Andratschke N, Wiedenmann N, Busch R, Grosu AL, Molls M: Radiotherapy for high-grade gliomas. Does altered fractionation improve the outcome? Strahlenther Onkol 2004, 180(7):401-407.

27. Nagy M, Schulz-Ertner D, Bischof M, Welzel T, Hof H, Debus J, Combs SE: Long-term outcome of postoperative irradiation in patients with newly diagnosed WHO grade III anaplastic gliomas. Tumori 2009, 95(3):317-324

28. Louis DN, Ohgaki H, Wiestler OD, Cavenee WK: Astrocytic tumors. In WHO classification of tumor of the central nervous system. 4th edition. Edited by Louis DN. Lyon: IARC Press; 2007:14-49.

29. Stupp R, Mason WP, van den Bent MJ, Weller M, Fisher B, Taphoorn MJ, Belanger K, Brandes AA, Marosi C, Bogdahn U, Curschmann J, Janzer RC, Ludwin SK, Gorlia T, Allgeier A, Lacombe D, Cairncross JG, Eisenhauer E, Mirimanoff RO, European Organisation for Research and Treatment of Cancer Brain Tumor and Radiotherapy Groups; National Cancer Institute of Canada Clinical Trials Group: Radiotherapy plus concomitant and adjuvant Temozolomide for glioblastoma. N Engl J Med 2005, 352(10):987-996.

30. Salazar-Onfray F, López MN, Mendoza-Naranjo A: Paradoxical effects of cytokines in tumor immune surveillance and tumor immune escape. Cytokine Growth Factor Rev 2007, 18(1-2):171-182.

31. Mumm JB, Oft M: Cytokine-based transformation of immune surveillance into tumor-promoting inflammation. Oncogene 2008, 27(45):5913-5919.

32. Barker FG, Davis RL, Chang SM, Prados MD: Necrosis as a prognostic factor in glioblastoma multiforme. Cancer 1996, 77(6):1161-1166.

33. Parsons DW, Jones S, Zhang $X$, Lin JC, Leary RJ, Angenendt P, Mankoo P, Carter H, Siu IM, Gallia GL, Olivi A, McLendon R, Rasheed BA, Keir S, Nikolskaya T, Nikolsky Y, Busam DA, Tekleab H, Diaz LA Jr, Hartigan J, Smith DR, Strausberg RL, Marie SK, Shinjo SM, Yan H, Riggins GJ, Bigner DD, Karchin R, Papadopoulos N, Parmigiani G, Vogelstein B, Velculescu VE, Kinzler KW: An integrated genomic analysis of human glioblastoma multiforme. Science 2008, 321(5897):1807-1812.

34. Pelloski CE, Lin E, Zhang L, Yung WK, Colman H, Liu JL, Woo SY, Heimberger AB, Suki D, Prados M, Chang S, Barker FG 3rd, Fuller GN, Aldape KD: Prognostic associations of activated mitogen-activated protein kinase and Akt pathways in glioblastoma. Clin Cancer Res 2006, 12(13):3935-3941.

doi:10.1186/1471-2407-13-247

Cite this article as: Xu et al.: The expression of cytoglobin as a prognostic factor in gliomas: a retrospective analysis of 88 patients. BMC Cancer 2013 13:247.

\section{Submit your next manuscript to BioMed Central and take full advantage of:}

- Convenient online submission

- Thorough peer review

- No space constraints or color figure charges

- Immediate publication on acceptance

- Inclusion in PubMed, CAS, Scopus and Google Scholar

- Research which is freely available for redistribution

Submit your manuscript at www.biomedcentral.com/submit 